\title{
Simple synthesis of nanosheets of rGO and nitrogenated rGO
}

\author{
Pallellappa Chithaiah ${ }^{1,2}$, Madhan Mohan Raju ${ }^{1}$, Giridhar U. Kulkarni ${ }^{1}$ and C. N. R. Rao ${ }^{* 2}$
}

\section{Full Research Paper}

Address:

${ }^{1}$ Centre for Nano and Soft Matter Sciences, Jalahalli, Bangalore, 560013, India and ${ }^{2} \mathrm{New}$ Chemistry Unit, International Centre for Materials Science, Jawaharlal Nehru Centre for Advanced Scientific Research, Jakkur P.O., Bangalore, 560064, India

Email:

C. N. R. Rao* - cnrrao@jncasr.ac.in

* Corresponding author

\section{Keywords:}

nanosheets; nitrogenated reduced graphene oxide (N-rGO); reduced graphene oxide ( $\mathrm{rGO}$ ); supercapacitors; thermal decomposition
Beilstein J. Nanotechnol. 2020, 11, 68-75. doi:10.3762/bjnano.11.7

Received: 12 October 2019

Accepted: 14 December 2019

Published: 07 January 2020

This article is part of the thematic issue "Graphene and beyond".

Associate Editor: A. Gölzhäuser

(c) 2020 Chithaiah et al.; licensee Beilstein-Institut. License and terms: see end of document.

\begin{abstract}
A green and facile approach has been developed for the large-scale synthesis of nanosheets of reduced graphene oxide (rGO) and nitrogenated reduced graphene oxide (N-rGO). This has been achieved by direct thermal decomposition of sucrose and glycine at $475{ }^{\circ} \mathrm{C}$ in ca. 7 minutes, respectively. The present protocols for synthesizing rGO and N-rGO are simple and environmentally friendly as we do not use any harmful reagents, metal catalysts and solvents. Along with that, this method offers an inexpensive route with high yields to prepare rGO with a high nitrogen content (20-25 atom \%). To further improve the properties of the synthesized rGO sheets, hydrogen treatment has been carried out to reduce the oxygen functional groups. Cyclic voltammograms and charge-discharge experiments have been carried out to understand the supercapacitor behavior of rGO and hydrogen treated (H-rGO) samples.
\end{abstract}

\section{Introduction}

Graphene, the one atom thick two-dimensional material of $\mathrm{sp}^{2}$ hybridized carbon atoms has attracted much attention after its discovery $[1,2]$. It is a fascinating material used in various applications owing to its excellent electrical, optical, mechanical and thermal properties [3-5]. It has a unique electronic structure with a linear dispersion of Dirac electrons. Graphene oxide (GO) and reduced graphene oxide (rGO) are chemically modified forms of graphene, which are extensively studied in the field of science and engineering. Reduced graphene oxide has attracted significant interest due to its similarities to pristine graphene. It behaves like a semimetal or a semiconductor and is therefore used in a variety of hybrid systems such as batteries [6], electrodes [7] and photodetectors [8].

In 1958, Hummer and Offeman developed a chemical method to synthesize graphene oxide by acid treatment of graphite [9]. The graphene oxide thus obtained contains oxygen functional groups (-CO-, - $\mathrm{COC}-$ ) on the surface and edges of the carbon sheet, which lead to a disruption of the conjugated network and the flow of charge carriers is reduced by several orders of mag- 
nitude [10]. Up to now, several methods including chemical vapor deposition [11-13], arc discharge [14], aerosol pyrolysis [15], mechanical exfoliation [1], solvothermal [16], hydrothermal synthesis [17], laser reduction of graphite oxide [18,19], and photo thermal deoxygenation of graphene oxide by camera flash have been developed to reduce the oxygen content of GO in order to restore the conjugated network [20]. Recently, a well-known chemical reduction method has been developed to obtain rGO through the reduction of exfoliated GO using various reducing agents such as hydrazine or dimethylhydrazine [21], $\mathrm{NaBH}_{4}$ [22], hydroquinone [23], or glucose [24]. However, these methods have not yet turned into a global strategy to prepare pure rGO in a scalable fashion. Therefore, searching for a new synthetic approach to obtain pure phases of rGO is a highly desirable and great challenge for materials chemists.

Herein, we report for the first time a generic and rapid method for the synthesis of rGO nanosheets by direct thermal decomposition of sugar, without the use of any solvents, metal catalysts, reagents and hazardous chemicals. Similarly, N-rGO nanosheets have also been synthesized using glycine as precursor.

\section{Results and Discussion}

The typical XRD patterns of rGO and N-rGO nanosheets are shown in Figure 1. The XRD pattern of the as-prepared rGO (Figure 1a) exhibits a broad peak at $23.5^{\circ}$ corresponding to an interlayer $d$-spacing of $0.378 \mathrm{~nm}$. The XRD pattern of N-rGO (Figure $1 b$ ) shows a diffraction peak at $25.8^{\circ}$ corresponding to an interlayer d spacing of $0.345 \mathrm{~nm}$. From the XRD patterns, it is observed that the peak commonly obtained for GO around $2 \theta$ of $10.3^{\circ}$ does not appear indicating that the precursors were directly converted into $\mathrm{rGO}$ and $\mathrm{N}$-rGO nanosheets.

Figure 2 shows the Raman spectra of the rGO and N-rGO nanosheets. The Raman spectrum of the rGO sample (Figure 2a) shows D, G and 2D band at, respectively, 1362, 1594 and $2880 \mathrm{~cm}^{-1}$. The spectrum of the N-rGO sample (Figure $2 \mathrm{~b}$ ) shows D, G and 2D band at, respectively, at 1354, 1581, and $2843 \mathrm{~cm}^{-1}$. The D-band is associated with the breathing modes of six-membered carbon rings that are activated by defects and structurally disordered, and the G-band originates from in-plane vibrations of $\mathrm{sp}^{2}$-hybridized carbon atoms in the rGO domains. The 2D-band is the second order of the D-band. The Raman results are consistent with previous reports [5].
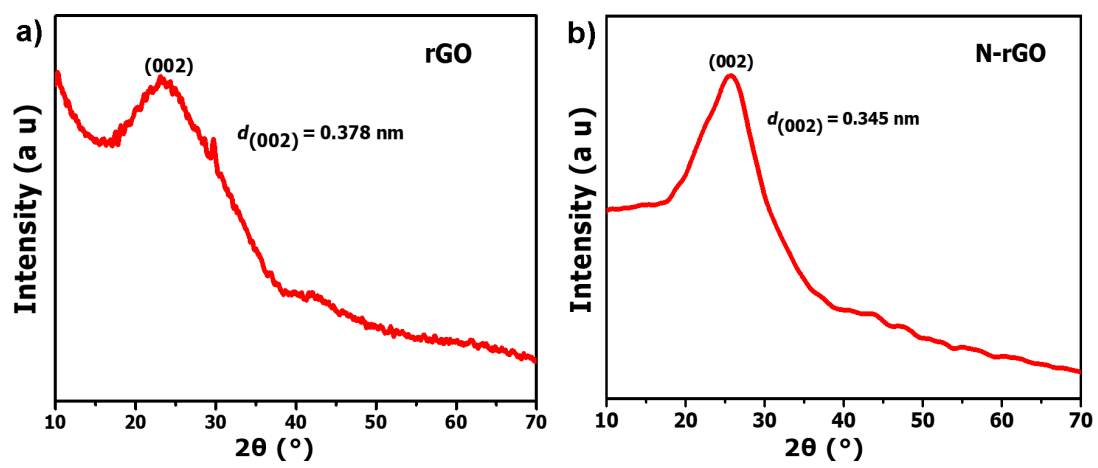

Figure 1: XRD patterns of (a) rGO and (b) N-rGO nanosheets.
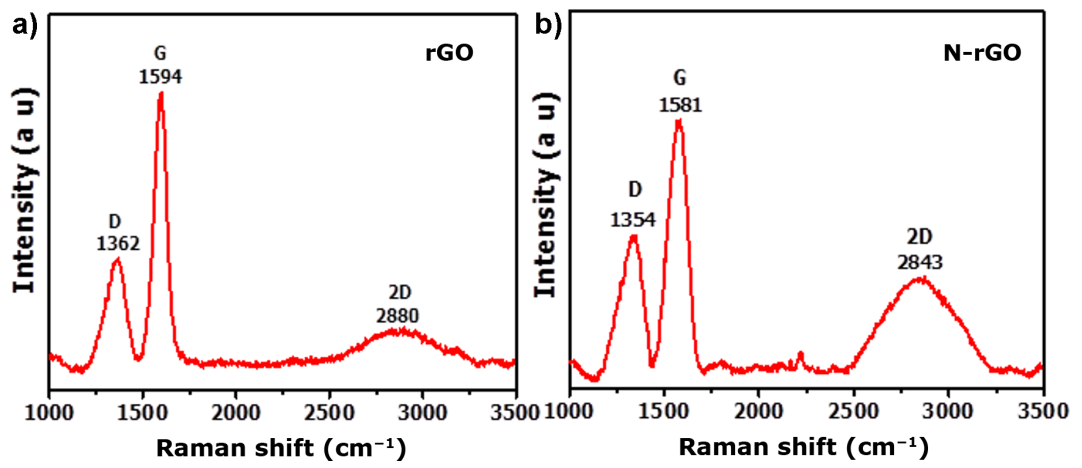

Figure 2: Raman spectra of (a) rGO and (b) N-rGO nanosheets. 
Thermogravimetric analysis (TGA) was carried out to investigate the thermal stability of the rGO and N-rGO nanosheets. The study was performed in an oxygen atmosphere at a heating rate of $3{ }^{\circ} \mathrm{C} \cdot \mathrm{min}^{-1}$. The results of $\mathrm{rGO}$ and $\mathrm{N}$-rGO are shown in Figure $3 \mathrm{a}$ and Figure $3 \mathrm{~b}$, respectively. The initial weight losses occurring for rGO and $\mathrm{N}-\mathrm{rGO}$ between room temperature and ca. $150{ }^{\circ} \mathrm{C}$ can be attributed to the evaporation of physically adsorbed water molecules.

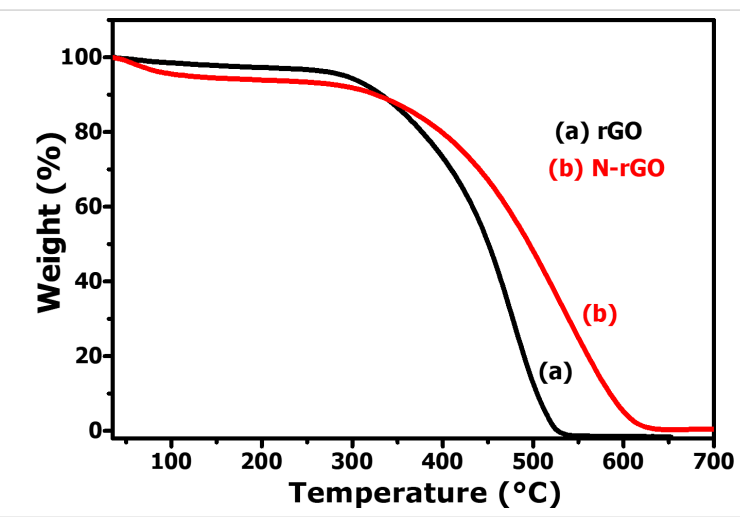

Figure 3: TGA curves of (a) rGO and (b) N-rGO nanosheets.

The second weight loss (93.4\%) for rGO occurring between 350 and $540{ }^{\circ} \mathrm{C}$ can be ascribed to the decomposition of the carbon network. For N-rGO nanosheets, a significant weight loss occurs in the temperature range between 400 and $625^{\circ} \mathrm{C}$, due to the decomposition of N-rGO. The results are consistent with the previous reports suggested in the literature $[25,26]$.

Typical SEM and TEM images of rGO at different magnifications are shown in Figure 4a,b. The low-magnification SEM image of the prepared rGO sample is composed of a large number of nanosheets, as shown in Figure 4a. The high-magnification SEM image (Figure 4b) shows that the nanosheets possess a smooth surface and are loosely stacked.

Energy-dispersive X-ray spectroscopy (EDS) was also measured to determine the chemical composition of rGO. Result from EDS shows (Figure 4c) that the product contains only $\mathrm{C}$ and $\mathrm{O}$. The atomic fractions of $\mathrm{C}$ and $\mathrm{O}$ are found to be $76.03 \%$ and $23.97 \%$, respectively, as shown in the inset of Figure 4c. The TEM image shown in Figure 4d indicates that the rGO sample is comprised of nanosheets with a smooth surface. The TEM image is in accordance with the SEM image (Figure $4 \mathrm{~b}$ ) of the sample. The selected area electron diffraction (SAED) pattern of the rGO sheets (inset in Figure 4d) shows a hexagonal pattern indicating the crystalline nature of the rGO sheets.

SEM images of N-rGO are shown in Figure 5a,b. The SEM images of N-rGO show morphological features that are similar
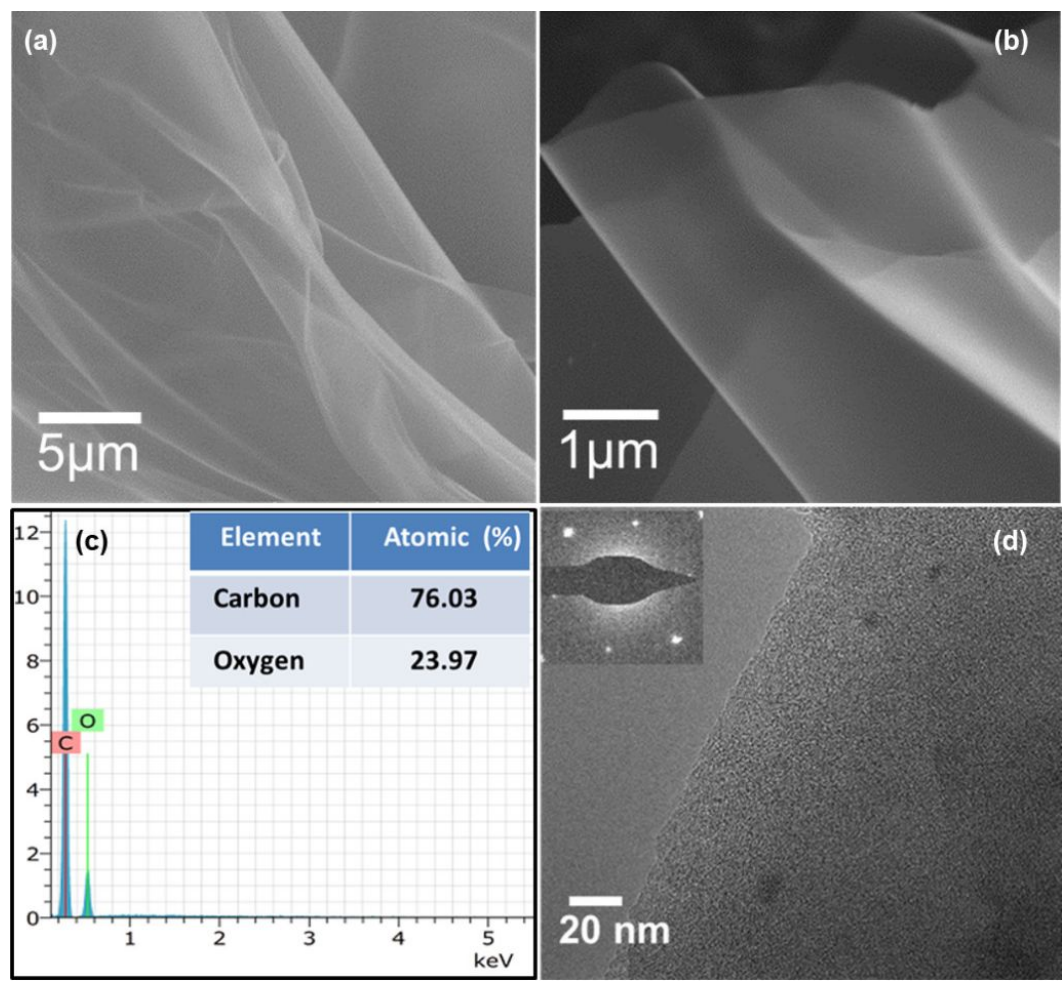

Figure 4: $(a, b)$ SEM images, (c) EDS pattern and chemical composition (inset), and (d) TEM image and SAED pattern (inset) of the rGO nanosheets. 
to those of rGO. EDS shows (Figure 5c) that the product contains only $\mathrm{C}, \mathrm{N}$ and $\mathrm{O}$. The atomic fractions of $\mathrm{C}, \mathrm{N}$ and $\mathrm{O}$ are found to be $66.26 \%, 21.94 \%$, and $11.81 \%$, respectively, as shown in the inset of Figure 5c. The presence of nitrogen in the as $\mathrm{N}$-rGO sample is also confirmed by the estimation of $\mathrm{N}$ content via CHNS analysis. The result shows that the weight percentage of $\mathrm{N}$ element in the N-rGO nanosheets is found to be approximately $20 \%$, and this is in good agreement with the EDS results.

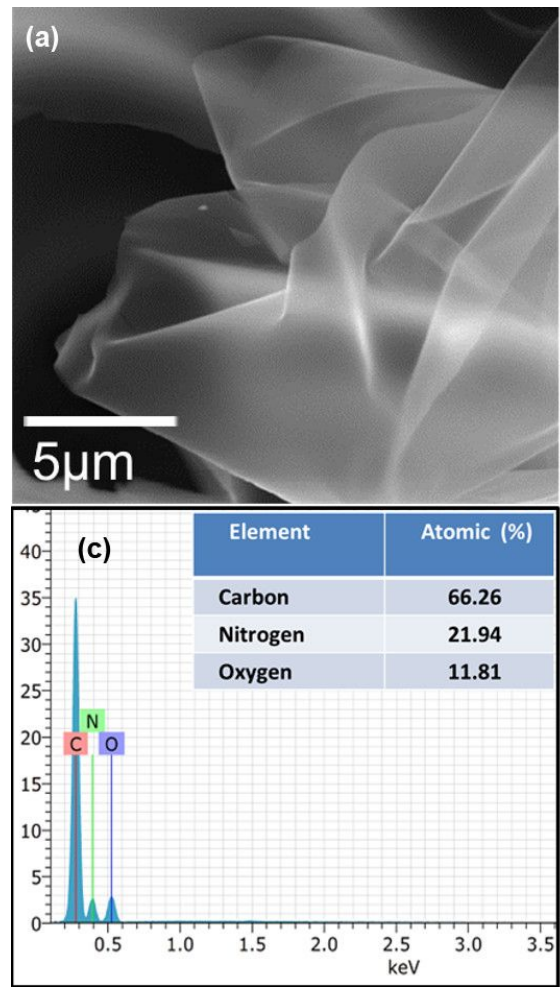

The TEM image in Figure 5d clearly shows that the N-rGO sample is composed of nanosheets with a smooth surface. The SAED pattern of the N-rGO sheet (inset in Figure 5d) shows a hexagonal pattern suggesting the crystalline nature of the synthesized N-rGO sheets.

AFM height images of as-prepared $\mathrm{rGO}$ and $\mathrm{N}-\mathrm{rGO}$ nanosheets are displayed in Figure $6 \mathrm{a}$ and Figure 6b, respectively. The rGO and N-rGO nanosheets are flat with an average thickness of

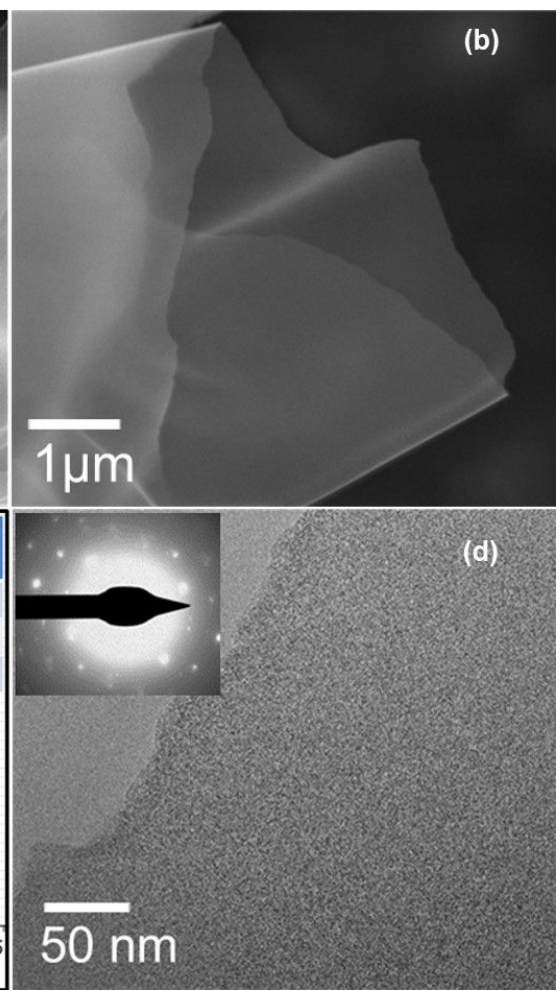

Figure 5: (a, b) SEM images, (c) EDS pattern and chemical composition (inset), and (d) TEM image and SAED pattern (inset) of the N-rGO nanosheets.
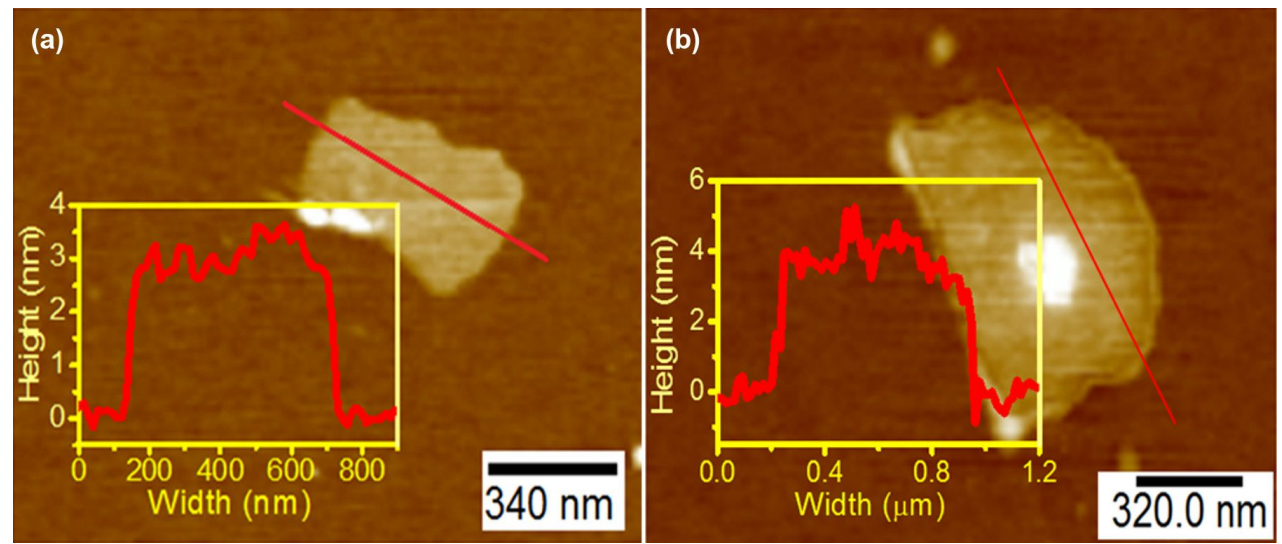

Figure 6: AFM images of (a) rGO and (b) N-rGO nanosheets. 
about $3 \mathrm{~nm}$ and $3.5 \mathrm{~nm}$, respectively, with their lateral dimension in the range of several hundred nanometers.

The experimental process and the digital photographs of rGO and N-rGO products are shown in Figure 7. Sucrose consists of one molecule of glucose and one molecule of fructose. The sucrose molecule contains - $\mathrm{OH},-\mathrm{CH}_{2} \mathrm{OH}$, and $-\mathrm{C}-\mathrm{O}-\mathrm{C}-$ functional groups. At a temperature of $475{ }^{\circ} \mathrm{C}$ in a pre-heated muffle furnace, within a short period of time, sucrose undergoes covalent cross-linking reactions yielding the formation of $\mathrm{C}-\mathrm{C}$ and

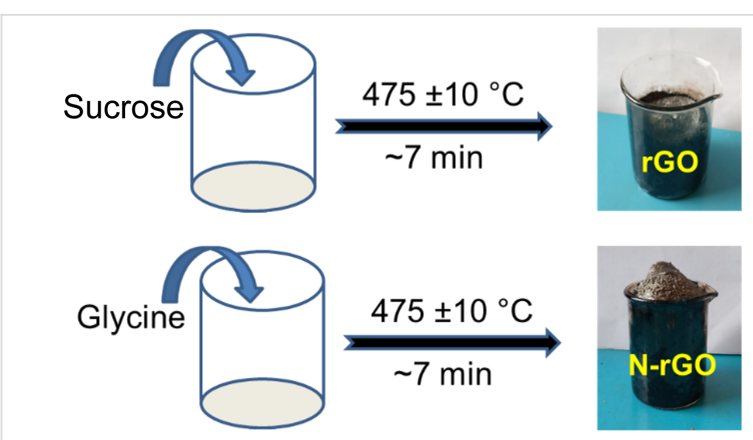

Figure 7: Schematic diagram of the formation of rGO and N-rGO nanosheets.
$\mathrm{C}=\mathrm{C}$ bonds through the removal of water and $\mathrm{CO}_{2}$. It is finally converted into a lightweight fluffy kind of material called graphene oxide nanosheets. The product was left in a furnace for ca. $7 \mathrm{~min}$ to get the pure phase without any impurities. The fraction of oxygen in the prepared sample is ca. 24 atom $\%$, as measured with EDS, and the XRD pattern of the sample shows a broad peak around $23.5^{\circ}$. Therefore, the obtained product can be considered to be reduced graphene oxide. Similarly, glycine also undergoes a cross-linking reaction with the removal of $\mathrm{CO}_{2}$ and $\mathrm{H}_{2} \mathrm{O}$ leading to the formation of nitrogen-doped reduced graphene oxide nanosheets. For comparison we have also synthesized rGO sheets at 400 and $600{ }^{\circ} \mathrm{C}$ as well. The corresponding XRD patterns, Raman spectra and SEM images are given in Figure S1 and Figure S2, respectively, in Supporting Information File 1.

In order to understand the supercapacitor behavior of rGO and H-rGO (hydrogen-treated rGO) samples, we have carried out cyclic voltammetry and $\mathrm{CD}$ experiments with three-electrode system using $1 \mathrm{M} \mathrm{H}_{2} \mathrm{SO}_{4}$ as electrolyte (Figure 8).

$\mathrm{CV}$ curves of rGO and $\mathrm{H}-\mathrm{rGO}$ at different scan rates from 5 to $200 \mathrm{mV} \cdot \mathrm{s}^{-1}$ vs $\mathrm{Hg} / \mathrm{Hg}_{2} \mathrm{SO}_{4}$ are shown in Figure $8 \mathrm{a}$ and
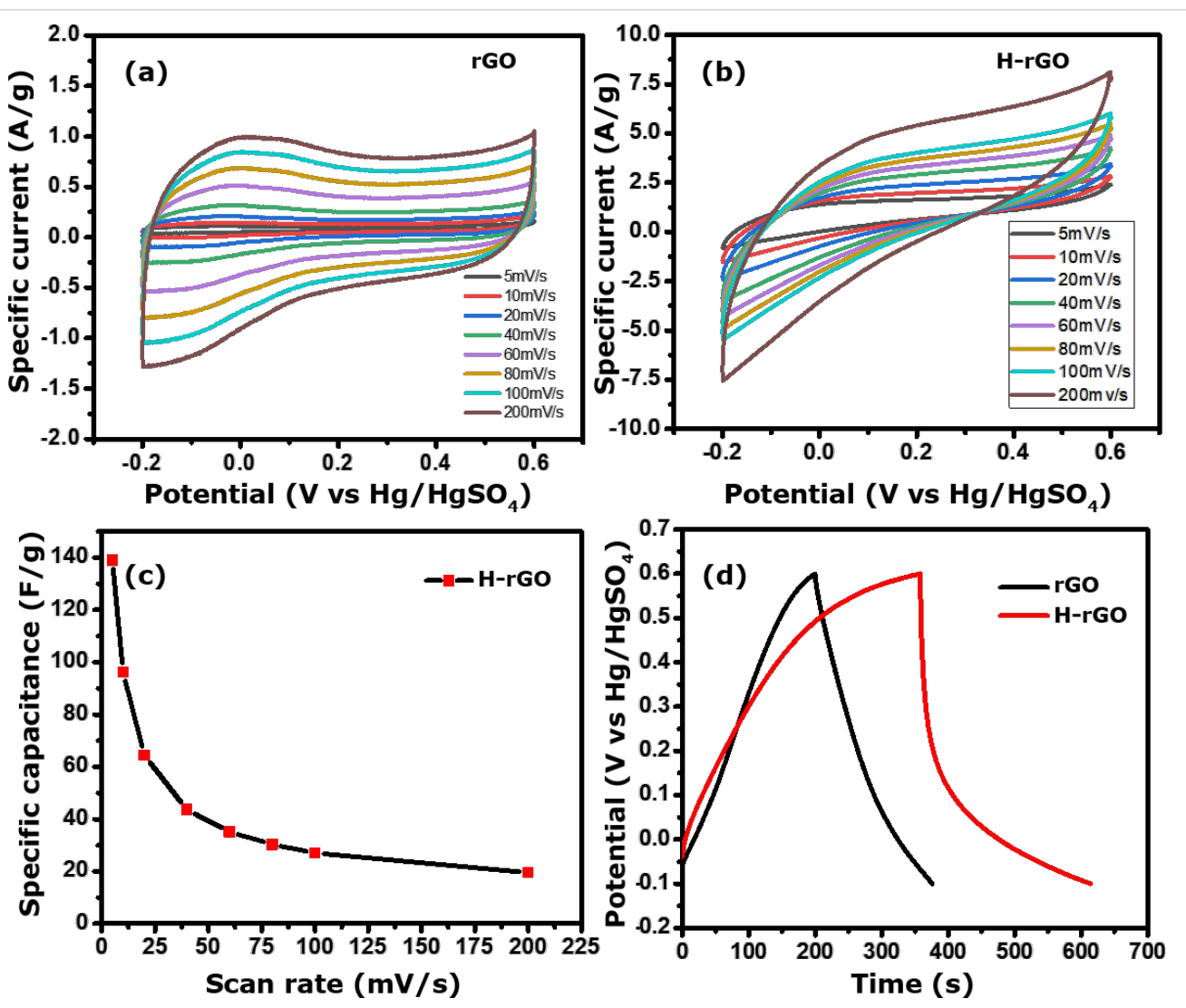

Figure 8: CV curves for (a) rGO and (b) H-rGO samples at different scan rates; (c) specific capacitance for H-rGO at scan rates from 5 to $200 \mathrm{mV} \cdot \mathrm{s}^{-1}$; (d) charge-discharge curves of rGO and $\mathrm{H}-\mathrm{rGO}$ at a current density of $0.5 \mathrm{~A} \cdot \mathrm{g}^{-1}$. 
Figure $8 \mathrm{~b}$, respectively. These curves show increase in current density with decreasing scan rate, suggesting that the samples have ideal capacitor characteristics. However, the H-rGO sample shows a higher current density and hence a higher specific capacitance than rGO. The calculated specific capacitance values from the $\mathrm{CV}$ of the $\mathrm{rGO}$ and $\mathrm{H}-\mathrm{rGO}$ electrodes at $5 \mathrm{mV} \cdot \mathrm{s}^{-1}$ are 7 (not shown) and $139 \mathrm{~F} \cdot \mathrm{g}^{-1}$, respectively. We have checked the rate capability of the working electrode at different scan rates of 10,20, 40,60,80,100, and $200 \mathrm{mV} \cdot \mathrm{s}^{-1}$ and observed that the specific capacitance values were 96, 64, 43, 35, 30, 27 and $19 \mathrm{~F} \cdot \mathrm{g}^{-1}$, respectively (Figure 8c). For comparison, the charge-discharge (CD) curves for rGO and H-rGO nanosheets were recorded at a current density of $0.5 \mathrm{~A} \cdot \mathrm{g}^{-1}$ in $1 \mathrm{M} \mathrm{H}_{2} \mathrm{SO}_{4}$ (Figure 8d). The specific capacitance values obtained from the $\mathrm{CD}$ curves at a current density of $0.5 \mathrm{~A} \cdot \mathrm{g}^{-1}$ for rGO and H-rGO were 137 and $203 \mathrm{~F} \cdot \mathrm{g}^{-1}$, respectively. The CD curve of H-rGO also shows a higher specific capacitance compared to rGO. The higher specific capacitance of H-rGO is attributed an increased conductivity due to the reduced number of functional groups after hydrogen reduction of the rGO sample. After 1000 cycles, the H-rGO sample shows $73 \%$ retention, implying that the H-rGO has excellent stability (Figure 9). We

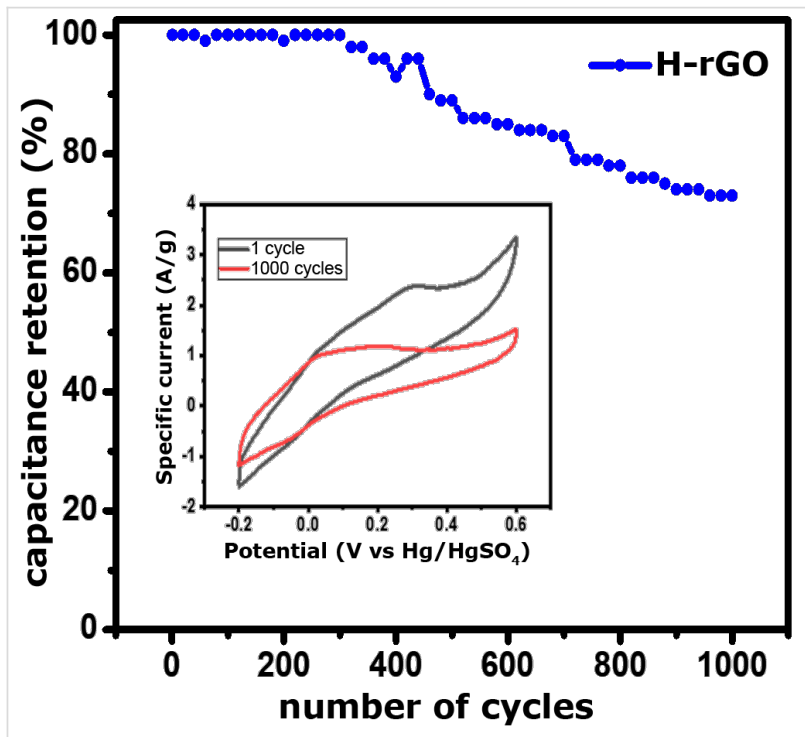

Figure 9: Cycling stability of H-rGO. compared our result with other materials reported recently (Table 1). For conductivity measurements, the H-rGO sample was dispersed in ethanol and drop-cast on a gold gap electrode. The average resistance measured using a Keithley source meter is ca. $4 \mathrm{M} \Omega$. The corresponding electrical conductivity obtained for $\mathrm{H}-\mathrm{rGO}$ is ca. $0.068 \mathrm{~S} / \mathrm{m}$.

\section{Conclusion}

In conclusion, we present a very simple and efficient method for the successful synthesis of nanosheets of rGO and of rGO with high nitrogen content by the thermal decomposition of sucrose and glycine. We measured the specific capacitance and carried out charge-discharge experiments. The rGO nanosheets that were hydrogen-treated (H-rGO) showed good supercapacitor behavior.

\section{Experimental}

\section{Preparation of rGO and N-rGO nanosheets}

Sucrose in the form of granulated table sugar from a retail store and analytical grade glycine from Sigma-Aldrich were purchased and used without further purification. Reduced graphene oxide (rGO) was prepared by using granulated table sugar. $2.0 \mathrm{~g}$ of sucrose was taken in a $100 \mathrm{~mL}$ borosil glass beaker. Then, the beaker was directly introduced into the preheated muffle furnace maintained at $475 \pm 10{ }^{\circ} \mathrm{C}$ in oxygen atmosphere. The sugar undergoes dehydration, producing a black foam in ca. 7 min. Finally, the resultant product was collected for further analysis. A similar procedure was followed to prepare $\mathrm{N}-\mathrm{rGO}$ using glycine as precursor.

\section{Hydrogen treatment of rGO nanosheets}

Hydrogen-treated reduced graphene oxide (H-rGO) was obtained as follows. $0.5 \mathrm{~g}$ of the rGO sample was taken in a ceramic boat and placed in a tube furnace. The sample was heated at $700{ }^{\circ} \mathrm{C}$ for $1 \mathrm{~h}$ in a continuous flow of $\mathrm{H}_{2} / \mathrm{Ar}$ gas (5\% hydrogen gas with Argon). After the reaction, the temperature was allowed to cool down to room temperature naturally. The resulting product was collected and used for the electrochemical supercapacitor measurements. The obtained results were compared with the as-synthesized rGO nanosheets.

Table 1: Comparison of the specific capacitance of H-rGO sheets with reported values.

\begin{tabular}{llll} 
material & structure & specific capacitance $\left(\mathrm{F} \cdot \mathrm{g}^{-1}\right)$ & reference \\
\hline porous electrochemically rGO & nanosheets & $81 \pm 3$ & {$[27]$} \\
graphene oxide & nanosheets & 121 & {$[28]$} \\
$\mathrm{RGO} / \mathrm{H}_{x} \mathrm{PO}_{y}$ & nanosheets & 101 & {$[29]$} \\
$\mathrm{RGO}$ & nanosheets & 90 & {$[29]$} \\
$\mathrm{H}-\mathrm{rGO}$ & nanosheets & 139 & this work
\end{tabular}




\section{Materials characterization}

The samples were characterized using transmission electron microscopy (TEM), atomic force microscopy (AFM), X-ray diffraction (XRD) and thermogravimetric analysis (TGA). $\mathrm{X}$-ray diffraction patterns of the samples were collected in the range of $10-70^{\circ}(2 \theta)$ using a Bruker D8 diffractometer with a $\mathrm{Cu} \mathrm{K} \alpha$ source $(\lambda=0.154178 \mathrm{~nm})$. The morphology of the samples was examined using a Tescan Mira3 field-emission scanning electron microscope (FESEM) equipped with an energydispersive X-ray spectroscopy (EDS). The TEM, HRTEM images and SAED patterns were obtained on a TALOS F200S G2, $200 \mathrm{kV}$ FEG, and a CMOS camera $(4 \mathrm{k} \times 4 \mathrm{k})$. The TEM samples were prepared by suspending the samples in ethanol, using an ultrasonic bath, and subsequent dripping of the suspension on the grid and drying. Raman spectra of the samples were recorded using a Jobin Yvon LabRam HR spectrometer with a $514 \mathrm{~nm}$ Ar laser. Thermogravimetric analysis of the samples was carried out in an oxygen flow with a heating rate of $3{ }^{\circ} \mathrm{C} \cdot \mathrm{min}^{-1}$ using a Mettler-Toledo-TG-850 apparatus. AFM measurements were performed using a $\mathrm{CP} 2$ atomic force microscope.

\section{Electrode preparation and electrochemical characterization}

The catalyst inks of as-synthesized rGO and reduced graphene oxide $\mathrm{H}-\mathrm{rGO}$ were prepared by ultrasonication separately. A mixture of $4.0 \mathrm{mg}$ rGO and $0.025 \mathrm{wt} \%(5 \mu \mathrm{L})$ of Nafion in $0.4 \mathrm{~mL}$ of dimethylformamide (DMF) was sonicated until a homogeneous dispersion was obtained. $3 \mu \mathrm{L}$ catalyst ink was taken and drop-cast onto a glassy carbon electrode, which was allowed to dry at room temperature. A similar procedure was followed to prepare the H-rGO electrode. The electrochemical studies, including cyclic voltammetry (CV) and chronopotentiometry charge-discharge (CD), were carried out at room temperature in $1 \mathrm{M} \mathrm{H}_{2} \mathrm{SO}_{4}$ solution in a standard three-electrode cell using an electrochemical workstation CHI 660E. This system consists of a glassy carbon working electrode $(3 \mathrm{mM})$, a platinum wire counter electrode and a $\mathrm{Hg} / \mathrm{Hg}_{2} \mathrm{SO}_{4}$ reference electrode with $1 \mathrm{M} \mathrm{H}_{2} \mathrm{SO}_{4}$ electrolyte. The specific capacitance (SC) of rGO and $\mathrm{H}-\mathrm{rGO}$ was calculated from $\mathrm{CV}$ curves, according to Equation 1:

$$
\mathrm{SC}=\int I \mathrm{~d} V / 2 v m \cdot \Delta V
$$

where $\int I \mathrm{~d} V$ is the area under the CV curve, $m$ is the mass of the active material, $v$ is the potential scan rate $(\mathrm{V} / \mathrm{s})$, and $\Delta V$ is the potential window. We have also calculated the specific capacitance from CD curves using Equation 2:

$$
\mathrm{SC}=I t / m \cdot \Delta V,
$$

where $I$ is the current, $t$ is the time of the discharge cycle, $m$ is the mass of the active material, and $\Delta V$ is the potential window of the discharge cycle.

\section{Supporting Information}

\section{Supporting Information File 1}

Additional experimental data.

[https://www.beilstein-journals.org/bjnano/content/

supplementary/2190-4286-11-7-S1.pdf]

\section{Acknowledgements}

PC thanks CeNS for fellowship and JNCASR for facilities. The authors acknowledge the TEM Facility, funded by TPF Nanomission, GoI project at CeNS, Bengaluru. The authors thank Bharath Bannur and Sunil Walia for the conductivity measurements.

\section{References}

1. Novoselov, K. S.; Geim, A. K.; Morozov, S. V.; Jiang, D.; Zhang, Y.; Dubonos, S. V.; Grigorieva, I. V.; Firsov, A. A. Science 2004, 306, 666-669. doi:10.1126/science.1102896

2. Novoselov, K. S.; Jiang, D.; Schedin, F.; Booth, T. J.; Khotkevich, V. V.; Morozov, S. V.; Geim, A. K. Proc. Natl. Acad. Sci. U. S. A. 2005, 102, 10451-10453. doi:10.1073/pnas.0502848102

3. Kumar, P. V.; Bardhan, N. M.; Tongay, S.; Wu, J.; Belcher, A. M.; Grossman, J. C. Nat. Chem. 2014, 6, 151-158. doi:10.1038/nchem.1820

4. Rao, C. N. R.; Sood, A. K.; Subrahmanyam, K. S.; Govindaraj, A. Angew. Chem., Int. Ed. 2009, 48, 7752-7777. doi:10.1002/anie.200901678

5. Rao, C. N. R.; Matte, H. S. S. R.; Subrahmanyam, K. S. Acc. Chem. Res. 2013, 46, 149-159. doi:10.1021/ar300033m

6. Zhu, X.; Zhu, Y.; Murali, S.; Stoller, M. D.; Ruoff, R. S. ACS Nano 2011, 5, 3333-3338. doi:10.1021/nn200493r

7. Kholmanov, I. N.; Domingues, S. H.; Chou, H.; Wang, X.; Tan, C.; Kim, J.-Y.; Li, H.; Piner, R.; Zarbin, A. J. G.; Ruoff, R. S. ACS Nano 2013, 7, 1811-1816. doi:10.1021/nn3060175

8. Abid; Sehrawat, P.; Islam, S. S.; Mishra, P.; Ahmad, S. Sci. Rep. 2018, 8, 3537. doi:10.1038/s41598-018-21686-2

9. Hummers, W. S., Jr.; Offeman, R. E. J. Am. Chem. Soc. 1958, 80, 1339. doi:10.1021/ja01539a017

10. Hossain, M. Z.; Johns, J. E.; Bevan, K. H.; Karmel, H. J.; Liang, Y. T.; Yoshimoto, S.; Mukai, K.; Koitaya, T.; Yoshinobu, J.; Kawai, M.; Lear, A. M.; Kesmodel, L. L.; Tait, S. L.; Hersam, M. C. Nat. Chem. 2012, 4, 305-309. doi:10.1038/nchem.1269

11. Kim, K. S.; Zhao, Y.; Jang, H.; Lee, S. Y.; Kim, J. M.; Kim, K. S.; Ahn, J.-H.; Kim, P.; Choi, J.-Y.; Hong, B. H. Nature 2009, 457, 706-710. doi:10.1038/nature07719

12. Subrahmanyam, K. S.; Vivekchand, S. R. C.; Govindaraj, A.; Rao, C. N. R. J. Mater. Chem. 2008, 18, 1517-1523. doi:10.1039/b716536f 
13. Li, X.; Cai, W.; An, J.; Kim, S.; Nah, J.; Yang, D.; Piner, R.; Velamakanni, A.; Jung, I.; Tutuc, E.; Banerjee, S. K.; Colombo, L.; Ruoff, R. S. Science 2009, 324, 1312-1314. doi:10.1126/science.1171245

14. Subrahmanyam, K. S.; Panchakarla, L. S.; Govindaraj, A.; Rao, C. N. R. J. Phys. Chem. C 2009, 113, 4257-4259. doi:10.1021/jp900791y

15. Campos-Delgado, J.; Romo-Herrera, J. M.; Jia, X.; Cullen, D. A.; Muramatsu, H.; Kim, Y. A.; Hayashi, T.; Ren, Z.; Smith, D. J.; Okuno, Y.; Ohba, T.; Kanoh, H.; Kaneko, K.; Endo, M.; Terrones, H.; Dresselhaus, M. S.; Terrones, M. Nano Lett. 2008, 8, 2773-2778. doi:10.1021/nl801316d

16. Choucair, M.; Thordarson, P.; Stride, J. A. Nat. Nanotechnol. 2009, 4, 30-33. doi:10.1038/nnano.2008.365

17. Huang, H.-H.; De Silva, K. K. H.; Kumara, G. R. A.; Yoshimura, M. Sci. Rep. 2018, 8, 6849. doi:10.1038/s41598-018-25194-1

18. Sokolov, D. A.; Shepperd, K. R.; Orlando, T. M. J. Phys. Chem. Lett. 2010, 1, 2633-2636. doi:10.1021/jz100790y

19. Kumar, P.; Subrahmanyam, K. S.; Rao, C. N. R. Int. J. Nanosci. 2011, 10, 559-566. doi:10.1142/s0219581×11008824

20. Gilje, S.; Dubin, S.; Badakhshan, A.; Farrar, J.; Danczyk, S. A.; Kaner, R. B. Adv. Mater. (Weinheim, Ger.) 2010, 22, 419-423. doi:10.1002/adma.200901902

21. Park, S.; An, J.; Jung, I.; Piner, R. D.; An, S. J.; Li, X.; Velamakanni, A.; Ruoff, R. S. Nano Lett. 2009, 9, 1593-1597. doi:10.1021/nl803798y

22. Shin, H.-J.; Kim, K. K.; Benayad, A.; Yoon, S.-M.; Park, H. K.; Jung, I.-S.; Jin, M. H.; Jeong, H.-K.; Kim, J. M.; Choi, J.-Y.; Lee, Y. H. Adv. Funct. Mater. 2009, 19, 1987-1992. doi:10.1002/adfm.200900167

23. Wang, G.; Yang, J.; Park, J.; Gou, X.; Wang, B.; Liu, H.; Yao, J. J. Phys. Chem. C 2008, 112, 8192-8195. doi:10.1021/jp710931h

24. Zhu, C.; Guo, S.; Fang, Y.; Dong, S. ACS Nano 2010, 4, 2429-2437. doi:10.1021/nn1002387

25. Park, O.-K.; Choi, Y.-M.; Hwang, J. Y.; Yang, C.-M.; Kim, T.-W.; You, N.-H.; Koo, H. Y.; Lee, J. H.; Ku, B.-C.; Goh, M. Nanotechnology 2013, 24, 185604. doi:10.1088/0957-4484/24/18/185604

26. Porwal, J.; Karanwal, N.; Kaul, S.; Jain, S. L. New J. Chem. 2016, 40, 1547-1553. doi:10.1039/c5nj02095f

27. Purkait, T.; Singh, G.; Kumar, D.; Singh, M.; Dey, R. S. Sci. Rep. 2018, 8, 640. doi:10.1038/s41598-017-18593-3

28. Down, M. P.; Rowley-Neale, S. J.; Smith, G. C.; Banks, C. E. ACS Appl. Energy Mater. 2018, 1, 707-714. doi:10.1021/acsaem.7b00164

29. Cao, J.; He, P.; Brent, J. R.; Yilmaz, H.; Lewis, D. J.; Kinloch, I. A.; Derby, B. ACS Appl. Mater. Interfaces 2018, 10, 10330-10338. doi:10.1021/acsami.7b18853

\section{License and Terms}

This is an Open Access article under the terms of the Creative Commons Attribution License (https://creativecommons.org/licenses/by/4.0). Please note that the reuse, redistribution and reproduction in particular requires that the authors and source are credited.

The license is subject to the Beilstein Journal of Nanotechnology terms and conditions: (https://www.beilstein-journals.org/bjnano)

The definitive version of this article is the electronic one which can be found at:

doi:10.3762/bjnano.11.7 